\title{
Rashba effect and magnetic field in quantum wires
}

\author{
S. Debald* and B. Kramer \\ 1. Institut für Theoretische Physik, Universität Hamburg, Jungiusstr. 9, 20355 Hamburg, Germany
}

(Dated: June 14, 2021)

\begin{abstract}
We investigate the influence of a perpendicular magnetic field on the spectral and spin properties of a ballistic quasi-one-dimensional electron system with Rashba effect. The magnetic field strongly alters the spin-orbit induced modification to the subband structure when the magnetic length becomes comparable to the lateral confinement. A new subband-dependent energy splitting at $k=0$ is found which can be much larger than the Zeeman splitting. This is due to the breaking of a combined spin orbital-parity symmetry.
\end{abstract}

PACS numbers: 85.75.-d, 73.23.Ad, 73.63.Nm, 71.70.Ej

\section{INTRODUCTION}

The quest for a better understanding of the influence of the electron spin on the charge transport in nonmagnetic semiconductor nanostructures has considerably attracted interest during recent years $\stackrel{1}{\frac{1}{1}}$ Spin-orbit interaction (SOI) is considered as a possibility to control and manipulate electron states via gate voltages ${ }^{2.3}$ This has generated considerable research activity, both in theory and experiment, motivated by fundamental physics as well as applicational aspects. Especially, SOI induced by the Rashba effect 4.5 in semiconductor heterostructures as a consequence of the lack of structure inversion symmetry ${ }^{6}$ is important. In these two-dimensional (2D) systems the Rashba effect leads to spin precession of the propagating electrons. The possibility to manipulate the strength of the Rashba effect by an external gate voltage has been demonstrated experimentally ${ }^{7.8 .9 .10}$ This is the basis of the spin dependent field-effect-transistor (spinFET) earlier discussed theoretically by Datta and Das. 11 Numerous theoretical spintronic devices have been proposed using interference, 12.13 .14 .15 resonant tunneling, 16,17,18,19 ferromagnet-semiconductor hybrid structures,$\underset{20,21,22,23}{ }$ multiterminal geometries, $, 24,25,26,27$ and adiabatic pumping 28 Magnetic field effects on the transport properties in 2D systems with SOI have been investigated theoretically ${ }^{29,30,31}$ as well as experimentally $7,8,9,10,32,33$.

In order to improve the efficiency of the spinFET the angular distribution of spin precessing electrons has to be restricted ${ }^{11}$ Thus, the interplay of SOI and quantum confinement in quasi-1D systems $34,35.36$ and quantum Hall edge channels ${ }^{37}$ has been studied. First experimental results on SOI in quantum wires have been obtained ${ }^{38}$ The presence of a perpendicular magnetic field has been suggested to relax the conditions for the external confining potential for quantum point contacts. In these systems a Zeeman-like spin splitting at $k=0$ has been predicted from the results of numerical calculations when simultaneously SOI and a magnetic field are present 39 The effect of an in-plane magnetic field on the electron transport in quasi-1D systems has also been calculated 40.41

In this work, we investigate the effect of a perpen- dicular magnetic field on the spectral and spin properties of a ballistic quantum wire with Rashba spin-orbit interaction. The results are twofold. First, we show that transforming the one-electron model to a bosonic representation yields a systematic insight into the effect of the SOI in quantum wires, by using similarities to atom-light interaction in quantum optics for high magnetic fields. Second, we demonstrate that spectral and spin properties can be systematically understood from the symmetry properties. Without magnetic field the system has a characteristic symmetry property - the invariance against a combined spin orbital-parity transformation - which is related to the presence of the SOI. This leads to the well-known degeneracy of energies at $k=0$. The eigenvalue of this symmetry transformation replaces the spin quantum number. A non-zero magnetic field breaks this symmetry and lifts the degeneracy. This magnetic field-induced energy splitting at $k=0$ can become much larger than the Zeeman splitting. In addition, we show that modifications of the one-electron spectrum due to the presence of the SOI are very sensitive to weak magnetic fields. Furthermore, we find characteristic hybridisation effects in the spin density. Both results are completely general as they are related to the breaking of the combined spin-parity symmetry.

This general argument explains the Zeeman-like splitting observed in recent numerical results $\frac{39}{39}$

\section{THE MODEL}

We study a quasi-1D quantum wire with SOI in a perpendicular magnetic field. The system is assumed to be generated in a $2 \mathrm{D}$ electron gas (2DEG) by means of a gate-voltage induced parabolic lateral confining potential. We assume that the SOI is dominated by structural inversion asymmetry. This is a reasonable approximation for InAs based 2DEGs 10 Therefore, the SOI is modelled by the Rashba Hamiltonian,, .5

$$
H=\frac{\left(\mathbf{p}+\frac{e}{c} \mathbf{A}\right)^{2}}{2 m}+V(x)+\frac{1}{2} g \mu_{\mathrm{B}} B \sigma_{z}-\frac{\alpha}{\hbar}\left[\left(\mathbf{p}+\frac{e}{c} \mathbf{A}\right) \times \boldsymbol{\sigma}\right]_{z},
$$




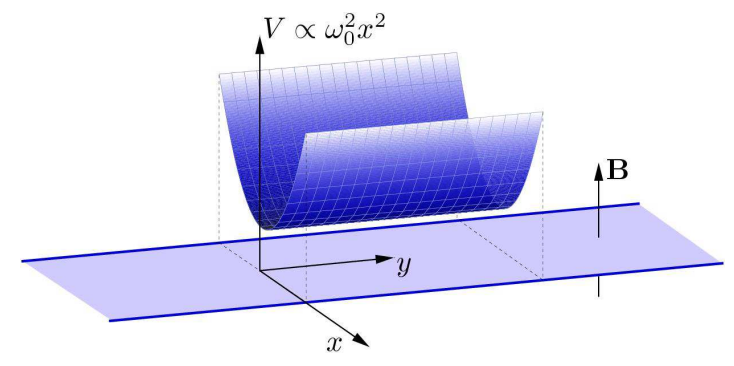

FIG. 1: (Color online) Model of the quantum wire.

where $m$ and $g$ are the effective mass and Landé factor of the electron, and $\boldsymbol{\sigma}$ is the vector of the Pauli matrices. The magnetic field is parallel to the $z$-direction (Fig. 团), and the vector potential $\mathbf{A}$ is in the Landau gauge. Three length scales characterise the relative strengths in the interplay of confinement, magnetic field $B$, and SOI,

$$
l_{0}=\sqrt{\frac{\hbar}{m \omega_{0}}}, \quad l_{B}=\sqrt{\frac{\hbar}{m \omega_{c}}}, \quad l_{\mathrm{SO}}=\frac{\hbar^{2}}{2 m \alpha} .
$$

The length scale $l_{0}$ corresponds to the confinement potential $V(x)=(m / 2) \omega_{0}^{2} x^{2}, l_{B}$ is the magnetic length with $\omega_{c}=e B / m c$ the cyclotron frequency and $l_{\mathrm{SO}}$ is the length scale associated with the SOI. In a 2DEG the latter is connected to a spin precession phase $\Delta \theta=L / l_{\mathrm{SO}}$ if the electron propagates a distance $L$.

Because of the translational invariance in the $y$ direction the eigenfunctions can be decomposed into a plain wave in the longitudinal direction and a spinor which depends only on the transversal coordinate $x$,

$$
\boldsymbol{\Psi}_{k}(x, y)=e^{i k y}\left(\begin{array}{c}
\phi_{k}^{\uparrow}(x) \\
\phi_{k}^{\downarrow}(x)
\end{array}\right)=: e^{i k y} \phi_{k}(x)
$$

With this and by defining creation and annihilation operators of a shifted harmonic oscillator, $a_{k}^{\dagger}$ and $a_{k}$, which describe the quasi-1D subbands in the case without SOI, the transversal wavefunction component satisfies

$$
H(k) \phi_{k}(x)=E_{k} \phi_{k}(x)
$$

for $k$ fixed with the Hamiltonian

$$
\begin{aligned}
\frac{H(k)}{\hbar \omega_{0}}= & \Omega\left(a_{k}^{\dagger} a_{k}+\frac{1}{2}\right)+\frac{1}{2} \frac{\left(k l_{0}\right)^{2}}{\Omega^{2}} \\
& +\frac{1}{2}\left(\begin{array}{c}
\xi_{1} k l_{0}+\xi_{2}\left(a_{k}+a_{k}^{\dagger}\right) \\
\xi_{3}\left(a_{k}-a_{k}^{\dagger}\right) \\
\delta
\end{array}\right) \cdot \boldsymbol{\sigma},
\end{aligned}
$$

the abbreviations

$$
\begin{gathered}
\Omega=\frac{\sqrt{\omega_{0}^{2}+\omega_{c}^{2}}}{\omega_{0}}=\sqrt{1+\left(\frac{l_{0}}{l_{B}}\right)^{4}}, \\
\xi_{1}=\frac{l_{0}}{l_{\mathrm{SO}}} \frac{1}{\Omega}, \\
\xi_{2}=\frac{1}{\sqrt{2}} \frac{l_{0}}{l_{\mathrm{SO}}}\left(\frac{l_{0}}{l_{B}}\right)^{2} \frac{1}{\sqrt{\Omega}}, \\
\xi_{3}=\frac{i}{\sqrt{2}} \frac{l_{0}}{l_{\mathrm{SO}}} \sqrt{\Omega},
\end{gathered}
$$

and the dimensionless Zeeman splitting

$$
\delta=\frac{1}{2}\left(\frac{l_{0}}{l_{B}}\right)^{2} \frac{m}{m_{0}} g
$$

( $m_{0}$ is the bare mass of the electron).

This representation of the Hamiltonian corresponds to expressing the transverse wavefunction in terms of oscillator eigenstates such that $a_{k}^{\dagger} a_{k}$ gives the subband index of the electron which propagate with longitudinal momentum $\hbar k$. The magnetic field leads to the lateral shift of the wavefunction and the renormalisation of the oscillator frequency $\Omega$. Moreover, the effective mass in the kinetic energy of the longitudinal propagation is changed. The last term in Eq. (5) describes how the SOI couples the electron's orbital degree of freedom to its spin. Due to the operators $a_{k}^{\dagger}$ and $a_{k}$ the subbands corresponding to one spin branch are coupled to the same and nearest neighboring subbands of opposite spin, see Fig. 22.

Formally, for $k$ fixed Eq. (5) can be regarded as a simple spin-boson system where the spin of the electron is coupled to a mono-energetic boson field which represents the transverse orbital subbands. This interpretation leads to an analogy to the atom-light interaction in quantum optics. There, the quantised bosonic radiation field is coupled to a pseudo-spin that approximates the two atomic levels between which electric dipol transitions occur. In our model, the roles of atomic pseudo-spin and light field are played by the spin and the orbital transverse modes of the electron, respectively.

Indeed, in the limit of a strong magnetic field, $l_{B} \ll$ $l_{0}$, and $k l_{0} \ll 1 \mathrm{Eq}$. (5) converges against the exactly integrable Jayne-Cummings model (JCM), 42

$$
\frac{H_{\mathrm{JC}}}{\hbar \omega_{c}}=a^{\dagger} a+\frac{1}{2}+\frac{1}{4} \frac{m}{m_{0}} g \sigma_{z}+\frac{1}{\sqrt{2}} \frac{l_{B}}{l_{\mathrm{SO}}}\left(a \sigma_{+}+a^{\dagger} \sigma_{-}\right) .
$$

This system is well known in quantum optics. It is one of the most simple models to couple a boson mode and a two-level system ${ }^{43}$ In the case of the quantum wire with SOI one can show that in the strong magnetic field limit the rotating-wave approximation, $\stackrel{43}{,}$ wich leads to the JCM, becomes exact. This is because for $l_{\mathrm{B}} \ll l_{0}$ and $k l_{0} \ll 1$ the electrons are strongly localised near the center of the quantum wire and thus insensitive to the confining potential. In this limit, there is a crossover to 
the $2 \mathrm{D}$ electron system with SOI in perpendicular magnetic field for which the formal identity to the JCM has been asserted previously ${ }^{44}$

In this context, it is important to note that the JCM is known to exhibit Rabi oscillations in optical systems with atomic pseudo-spin and light field periodically exchanging excitations. Recently, an experimentally feasible scheme for the production of coherent oscillations in a single few-electron quantum dot with SOI has been proposed 45 with the electron's spin and orbital angular momentum exchanging excitation energy. This highlights the general usefulness of mapping parabolically confined systems with SOI onto a bosonic representation as shown in Eq. (5). Related results have been found in a 3D model in nuclear physics where the SOI leads to a spin-orbit pendulum effect $\underline{46}$

\section{SYMMETRY PROPERTIES}

Without magnetic field it has been pointed out previously that one effect of SOI in 2D is that no common axis of spin quantisation can be found, see e.g. Ref ${ }^{36}$. Since the SOI is proportional to the momentum it lifts spin degeneracy only for $k \neq 0$. From the degeneracy at $k=0$ a new binary quantum number can be expected at $B=0$. It can easily be shown that for any symmetric confinement potential $V(x)=V(-x)$ in Eq. (1) - which includes the $2 \mathrm{D}$ case for $V \equiv 0$ or symmetric multi-terminal junctions $24.25,26$ - the Hamiltonian is invariant under the unitary transformation

$$
U_{x}=e^{i 2 \pi \hat{P}_{x} \hat{S}_{x} / \hbar}=i \hat{P}_{x} \sigma_{x}
$$

where $\hat{P}_{x}$ is the inversion operator for the $x$-component, $\hat{P}_{x} f(x, y)=f(-x, y)$. Thus, the observables $H, p_{y}$ and $\hat{P}_{x} \sigma_{x}$ commute pairwise. Without SOI, $\hat{P}_{x}$ and $\sigma_{x}$ are conserved separately. With SOI, both operators are combined to form the new constant of motion $\hat{P}_{x} \sigma_{x}$ which is called spin parity. When introducing a magnetic field with a non-zero perpendicular component the spin parity symmetry is broken and we expect the degeneracy at $k=0$ to be lifted. As a side remark, by using oscillator eigenstates and the representation of eigenstates of $\sigma_{z}$ for the spinor, the Hamiltonian $H(k)$ in Eq. (5) becomes real and symmetric. We point out that in this choice of basis the transformation $U_{y}=i \hat{P}_{y} \sigma_{y}$ is a representation of the time-reversal operation which for $B=0$ also commutes with $H$. However, it does not commute with $p_{y}$ and no further quantum number can be derived from $U_{y} \stackrel{47}{\underline{47}}$ The effect of the symmetry $\hat{P}_{x} \sigma_{x}$ on the transmission through symmetric four ${ }^{24}$ and three-terminal ${ }^{25}$ devices has been studied previously.

We recall that the orbital effect of the magnetic field leads to a twofold symmetry breaking: the breaking of the spin parity $\hat{P}_{x} \sigma_{x}$ lifts the $k=0$ degeneracy (even without the Zeeman effect) and the breaking of timereversal symmetry lifts the Kramers degeneracy. For
$B=0$ we can attribute the quantum numbers $(k, n, s)$ to an eigenstate where $n$ is the subband index corresponding to the quantisation of motion in $x$-direction and $s= \pm 1$ is the quantum number of spin parity. For $B \neq 0$, due to the breaking of spin parity, $n$ and $s$ merge into a new quantum number leading to the nonconstant energy splitting at $k=0$ which will be addressed in the next Section when treating the spectral properties.

For weak SOI $\left(l_{\mathrm{SO}} \gg l_{0}\right)$ one finds in second order that the spin splitting at $k=0$ for the $n$th subband is

$$
\frac{\Delta_{n}}{\hbar \omega_{0}}=\delta+\frac{1}{2}\left(\frac{l_{0}}{l_{\mathrm{SO}}}\right)^{2} \frac{(\Omega-\delta) \chi_{1}^{2}-(\Omega+\delta) \chi_{2}^{2}}{\Omega^{2}-\delta^{2}}\left(n+\frac{1}{2}\right),
$$

where $\chi_{1,2}=2^{-1 / 2}\left[\left(l_{0} / l_{B}\right)^{2} \Omega^{-1 / 2} \mp \Omega^{1 / 2}\right]$. The first term is the bare Zeeman splitting and the SOI-induced second contribution has the peculiar property of being proportional to the subband index. In addition, for weak magnetic field $\left(l_{0} \ll l_{B}\right)$ the splitting is proportional $B$,

$$
\frac{\Delta_{n}}{\hbar \omega_{0}} \approx \delta-\left(\frac{l_{0}}{l_{\mathrm{SO}}}\right)^{2}\left(\frac{l_{0}}{l_{B}}\right)^{2}\left(1+\frac{1}{4} \frac{m}{m_{0}} g\right)\left(n+\frac{1}{2}\right) .
$$

This is expected because by breaking the spin parity symmetry at non-zero $B$ the formerly degenerate levels can be regarded as a coupled two-level system for which it is known that the splitting into hybridised energies is proportional to the coupling, i.e. the magnetic field $B$.

\section{SPECTRAL PROPERTIES}

Due to the complexity of the coupling between spin and subbands in Eq. (5), apart from some trivial limits, no analytic solution of the Schrödinger equation can be expected. We find the eigenfunctions and energies of the Hamiltonian by exact numerical diagonalisation. Figures $2 \mathrm{~b} \ldots \mathrm{d}$ show the spectra for different strengths of magnetic field and parameters typical for InAs: $\alpha=1.0 \cdot 10^{-11} \mathrm{eVm}, g=-8, m=0.04 m_{0}$. We set $l_{\mathrm{SO}}=l_{0}$ which corresponds to a wire width $l_{0} \approx 100 \mathrm{~nm}$.

For the case without magnetic field it has been asserted previously that the interplay of SOI and confinement leads to strong spectral changes like nonparabolicities and anticrossings when $l_{\mathrm{SO}}$ becomes comparable to $l_{0}{ }^{34,36}$ In Fig. 20 we find similar results in the limit of a weak magnetic field. However, as an effect of non-vanishing magnetic field we observe a splitting of the formerly spin degenerate energies at $k=0$. For the Zeeman effect it is expected that the corresponding spin splitting is constant. In contrast, in Fig. 2 2 ... d the splitting at $k=0$ depends on the subband. This additional splitting has been predicted in Sec. IIII in terms of a symmetry breaking effect when SOI and perpendicular magnetic field are simultaneously present.

Figure 3 shows the eigenenergies $E_{n}(k=0)$ for $l_{\mathrm{SO}}=$ $l_{0}$ as a function of magnetic field in units of hybridised energies, $\left(\omega_{0}^{2}+\omega_{c}^{2}\right)^{1 / 2}$. Three different regimes can be 
(a)

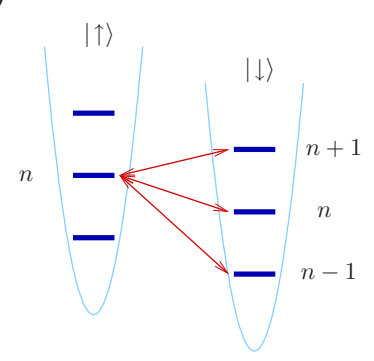

(c)

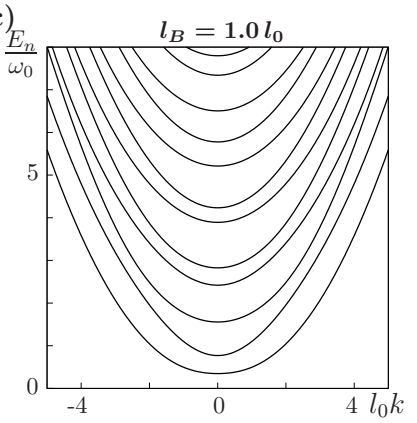

(b)

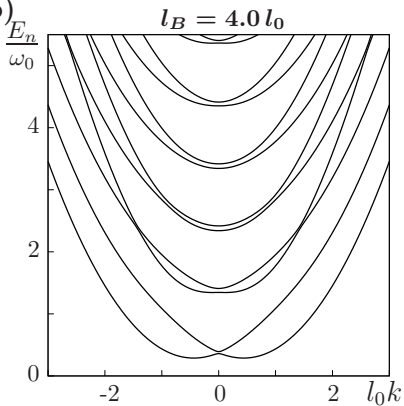

(d)

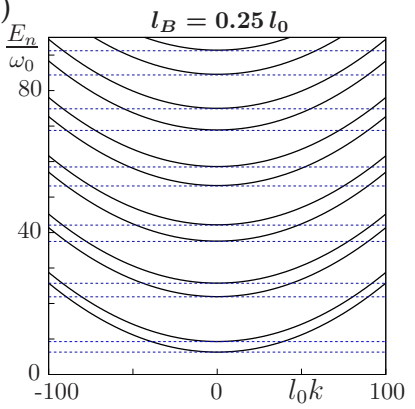

FIG. 2: (Color online) (a) Spin-orbit induced coupling of subbands with opposite spins in a quantum wire. (b)-(d) Spectra of a quantum wire with SOI for different strengths of perpendicular magnetic field for $l_{\mathrm{SO}}=l_{0}$ and typical InAs parameters: $\alpha=1.0 \cdot 10^{-11} \mathrm{eVm}, g=-8, m=0.04 m_{0}$. For strong magnetic field (d) the convergence towards the JaynesCummings model (JCM) can be seen (dashed: eigenenergies of JCM).

distinguished. (i) For small magnetic field $\left(l_{0} / l_{B} \ll 1\right)$ the energy splitting evolves from the spin degenerate case (triangles) due to the breaking of spin parity. Although the perturbative results Eq. (13) cannot be applied to the case $l_{\mathrm{SO}}=l_{0}$ in Fig. B $\mathrm{k}$, the energy splitting at small magnetic field and the overall increasing separation for higher subbands are reminiscent of the linear dependences on $n$ and $B$ found in Eqs. (13) and (14). (ii) For $l_{0} / l_{B} \approx 1$, the energy splitting is comparable to the subband separation which indicates the merging of the quantum numbers of the subband and the spin parity into a new major quantum number. For higher subbands, the SOI-induced splitting even leads to anticrossings with neighboring subbands. (iii) Finally, the convergence to the JCM implies that the splittings should saturate for large $B$ (Fig. 221 ). The dashed lines in Fig. 3a show the energies of the spin-split Landau levels, $E_{n} / \hbar \omega_{0}=\left(l_{0} / l_{B}\right)^{2}(n+1 / 2) \pm \delta / 2$ for $l_{0}=4 l_{B}$, indicating that the SOI-induced energy splitting is always larger than the bulk Zeeman splitting. At $l_{0} \approx l_{B}$ the SOIinduced splitting exceeds the Zeeman effect by a factor 5. This is remarkable because of the large value of the $g$-factor in InAs.

For our wire parameters the sweep in Fig. 3 corresponds to a magnetic field $B \approx 0 \ldots 1 \mathrm{~T}$. Considering

the significant spectral changes due to breaking of spin parity at $l_{B} \approx l_{0}(B \approx 70 \mathrm{mT})$ we conclude that the SOI-induced modifications in the wire subband structure are very sensitive to weak magnetic fields (Fig. 2 2 , c). This may have consequences for spinFET designs that rely on spin polarised injection from ferromagnetic leads because stray fields can be expected to alter the transmission probabilities of the interface region.

The SOI-induced enhancement of the spin splitting should be accessable via optical resonance or ballistic transport experiments. In a quasi-1D constriction the conductance is quantised in units of $n e^{2} / h$ where $n$ is the number of transmitting channels $\frac{48}{}$ In the following, we neglect the influence of the geometrical shape of the constriction and that for small magnetic fields $\left(l_{0} / l_{B}<0.5\right)$ the minima of the lowest subbands are not located at $k=0$ (Fig. 2 b). In this simplified model we expect the conductance $G$ to jump up one conductance quantum every time the Fermi energy passes through the minimum of a subband. Thus, in the case of spin degenerate subbands, $G$ increases in steps with heights $2 e^{2} / h$ (triangles in Fig. 3b).

In principle, by sweeping the magnetic field the different regimes discussed in Fig. 3. can be distinguished in the ballistic conductance. For high magnetic field, the spin degeneracy is broken due to the Zeeman effect. This leads to a sequence of large steps (Landau level separation) interrupted by small steps (spin splitting) (circles in Fig. 3b). As a signature of the SOI we expect increasing spin splitting for higher Landau levels due to converging towards the JCM (Fig. 22 $\mathrm{d}$ ). Decreasing the magnetic field enhances the effects of SOI until at $l_{B} \approx l_{0}$ subband and spin splitting are comparable whereas the Zeeman effect becomes negligible (crosses in Fig. Bb).

\section{SPIN PROPERTIES}

Not only the energy spectra of the quantum wire are strongly affected by the breaking the spin parity $\hat{P}_{x} \sigma_{x}$. The latter symmetry has also profound consequences for the spin density,

$$
\mathbf{S}_{n, k}(x):=\psi_{n, k}^{\dagger} \boldsymbol{\sigma} \psi_{n, k} .
$$

To elucidate this in some detail we start with considering the case $B=0$.

\section{A. Vanishing magnetic field}

Without magnetic field, the spin parity is a constant of motion. The corresponding symmetry operation Eq. (12) leads to the symmetry property for the wavefunction,

$$
\psi_{n, k, s}^{\uparrow}(x)=s \psi_{n, k, s}^{\downarrow}(-x), \quad s= \pm 1,
$$

where $s$ denotes the quantum number of the spin parity. This symmetry requires the spin density components 
(a)

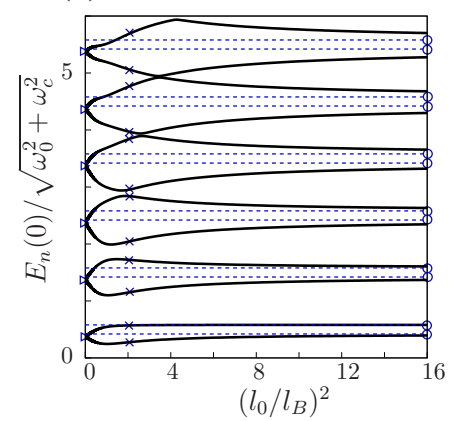

(b)

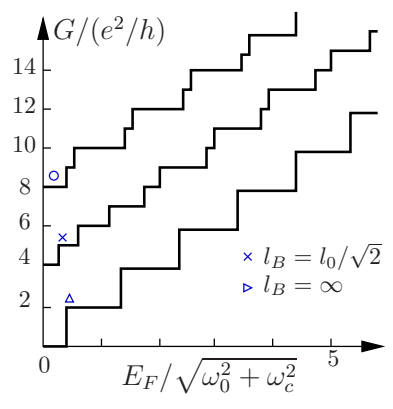

FIG. 3: (Color online) (a) Magnetic field evolution of $E_{n}(k=$ $0)$ for $l_{\mathrm{SO}}=l_{0}$ in units of $\sqrt{\omega_{0}^{2}+\omega_{c}^{2}}$. Three different regimes of spin splitting can be distinguished (see text). Dashed lines correspond to Zeeman split Landau levels. (b) Simplified sketch of the ballistic conductance as a function of Fermi energy for $\left(l_{0} / l_{B}\right)^{2}=0$ (triangles) and $\left(l_{0} / l_{B}\right)^{2}=2$ (crosses) (curves vertically shifted for clarity).

perpendicular to the confinement to be antisymmetric, $S_{n, k, s}^{y, z}(x)=-S_{n, k, s}^{y, z}(-x)$, leading to vanishing spin expectation values, $\left\langle\sigma_{y, z}\right\rangle_{n, k, s}=\int d x S_{n, k, s}^{y, z}(x)=0$. We note that using the $\sigma_{z}$-representation for spinors even leads to zero longitudinal spin density $S_{n, k, s}^{y}(x) \equiv 0$ because the real and symmetric Hamiltonian $H(k)$ implies real transverse wavefunctions independent of the spin parity. Therefore, it is sufficient to consider the $x$ - and $z$ components of the spin, only.

For zero magnetic field, it has been pointed out that for large $k$ the spin is approximately quantised in the confinement direction 36 This is due to the so-called longitudinalSOI approximation $\frac{35}{5}$ which becomes valid when the term linear in $k$ in the SOI [Eq. [5] exceeds the coupling to the neighboring subbands.

\section{B. Non-vanishing magnetic field}

The perpendicular magnetic field breaks spin parity and thereby leads to a hybridisation of formerly degenerate states for small $k$. In addition, the breaking of the symmetry of the wavefunction Eq. (16) leads to modifications of the spin density.

In Fig. 4 the expectation value of spin is shown as a function of the longitudinal momentum for the two lowest subbands. For weak magnetic field (Fig. 目) results similar to the zero magnetic field case ${ }^{36}$ are found. For large $k$ the spinor is effectively described by eigenstates of $\sigma_{x}$ which concurs with the longitudinal-SOI approximation. However, for $k \approx 0$ the hybridisation of the wavefunction leads to a finite value of $\left\langle\sigma_{z}\right\rangle$. This corresponds to the emergence of the energy splitting at $k=0$ in Fig. 20 which can be regarded as an additional effective Zeeman splitting that tilts the spin into the $\sigma_{z}$-direction - even without a real Zeeman effect. This effect becomes even

(a)

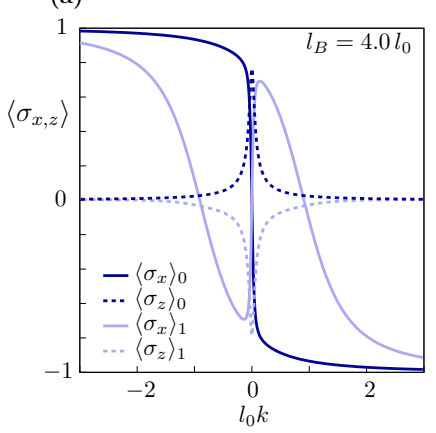

(b)

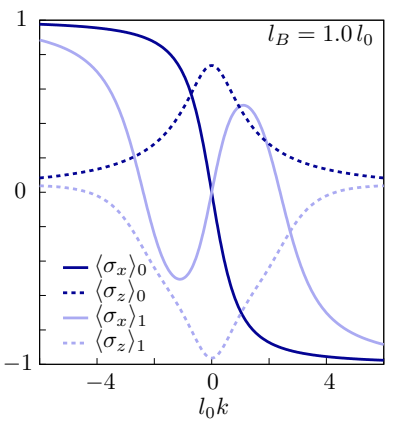

FIG. 4: Expectation values of spin for the two lowest eigenstates for $l_{\mathrm{SO}}=l_{0}$. Solid: $\left\langle\sigma_{x}\right\rangle_{n}$, dashed: $\left\langle\sigma_{z}\right\rangle_{n}$. (a) weak magnetic field, $l_{B}=4.0 l_{0}$. Hybridisation of wavefunction at $k \approx 0$ leads to finite $\left\langle\sigma_{z}\right\rangle_{n}$ component. (b) Strong magnetic field, $l_{B}=1.0 l_{0}$.

more pronounced for large magnetic field (Fig. [4). Here, for small $k$, the spin of the lowest subband is approximately quantised in $\sigma_{z}$ direction. The spin expectation values in Fig. 团 depend only marginally on the strength of the Zeeman effect. No qualitative difference is found for $g=0$.

\section{CONCLUSION}

In summary, the effect of a perpendicular magnetic field on a quasi-1D electron system with Rashba effect is investigated. It is shown that the spectral and spin features of the system for small $k$ are governed by a compound spin orbital-parity symmetry of the wire. Without magnetic field this spin parity is a characteristic property of symmetrically confined systems with Rashba effect and leads to a new binary quantum number which replaces the quantum number of spin. This symmetry is also responsible for the well-known degeneracy for $k=0$ in systems with Rashba effect. A non-zero magnetic field breaks the spin-parity symmetry and lifts the corresponding degeneracy, thus leading to a novel magnetic field induced energy splitting at $k=0$ which can become much larger than the Zeeman splitting. Moreover, we find that the breaking of the symmetry leads to hybridisation effects in the spin density.

The one-electron spectrum is shown to be very sensitive to weak magnetic fields. Spin-orbit interaction induced modifications of the subband structure are strongly changed when the magnetic length becomes comparable to the lateral confinement of the wire. This might lead to consequences for spinFET designs which depend on spin injection from ferromagnetic leads because of magnetic stray fields.

For the example of a quantum wire, we demonstrate that in the case of a parabolical confinement it is useful to map the underlying one-electron model onto a bosonic representation which shows for large magnetic field many 
similarities to the atom-light interaction in quantum optics. In Ref $\frac{45}{5}$ this mapping is utilised to predict spinorbit driven coherent oscillations in single quantum dots.

\section{ACKNOWLEDGEMENTS}

This work was supported by the EU via TMR and RTN projects FMRX-CT98-0180 and HPRN-CT2000-
0144, and DFG projects Kr 627/9-1, Br 1528/4-1. We are grateful to T. Brandes, T. Matsuyama and T. Ohtsuki for useful discussions.
* Electronic address: debald@physnet.uni-hamburg.de

1 I. Žutić, J. Fabian, and S. Das Sarma, Rev. Mod. Phys. 76, 323 (2004).

2 J. C. Egues, G. Burkard, and D. Loss, Phys. Rev. Lett. 89, 176401 (2002).

3 E. I. Rashba and Al. L. Efros, Phys. Rev. Lett. 91, 126405 (2003).

4 E. I. Rashba, Fiz. Tverd. Tela (Leningrad) 2, 1224 (1960) [Sov. Phys. Solid 2, 1109 (1960)].

5 Y. A. Bychkov and E. I. Rashba, Pis'ma Zh. Éksp. Teor. Fiz. 39, 66 (1984) [JETP Lett. 39, 78 (1984)].

${ }^{6}$ R. Winkler, Spin-Orbit Coupling Effect is Two-Dimensional Electron and Hole Systems (Springer, Berlin, 2003), Vol. 191 of Springer Tracts in Modern Physics.

7 J. Nitta, T. Akazaki, H. Takayanagi, and T. Enoki, Phys. Rev. Lett. 78, 1335 (1997).

8 G. Engels, J. Lange, T. Schäpers, and H. Lüth, Phys. Rev. B 55, R1958 (1997).

9 D. Grundler, Phys. Rev. Lett. 84, 6074 (2000).

10 T. Koga, J. Nitta, T. Akazaki, and H. Takayanagi, Phys. Rev. Lett. 89, 046801 (2002).

11 S. Datta and B. Das, Appl. Phys. Lett. 56, 665 (1990).

12 J. Nitta, F. E. Meijer, and H. Takayanagi, Appl. Phys. Lett. 75, 695 (1999).

13 X. F. Wang, P. Vasilopoulos, and F. M. Peeters, Appl. Phys. Lett. 80, 1400 (2002); Phys. Rev. B 65, 165217 (2002).

14 X. F. Wang and P. Vasilopoulos, Phys. Rev. B 68, 035305 (2003).

15 U. Zülicke, Appl. Phys. Lett. 85, 2616 (2004).

16 E. A. de Andrada e Silva and G. C. La Rocca, Phys. Rev. B 59, R15583 (1999).

17 T. Koga, J. Nitta, H. Takayanagi, and S. Datta, Phys. Rev. Lett. 88, 126601 (2002).

18 M. Governale, B. Boese, U. Zülicke, and C. Schroll, Phys. Rev. B 65, R140403 (2002).

19 R. Ionicioiu and I. D'Amico, Phys. Rev. B 67, R041307, (2003).

20 C.-M. Hu and T. Matsuyama, Phys. Rev. Lett. 87, 066803 (2001); T. Matsuyama et al., Phys. Rev. B 65, 155322 (2002).

21 M. H. Larsen, A. M. Lunde, and K. Flensberg, Phys. Rev. B 66, 033304 (2002).

${ }^{22}$ F. Mireles and G. Kirczenow, Phys. Rev. B 66, 214415 (2002).

23 M. G. Pala et al., Phys. Rev. B 69, 045304 (2004).

${ }^{24}$ E. N. Bulgakov et al., Phys. Rev. Lett. 83, 376 (1999).
25 A. A. Kiselev and K. W. Kim, Appl. Phys. Lett. 78, 775 (2001); ibid. 94, 4001 (2003).

26 T. P. Pareek, Phys. Rev. Lett. 92, 076601 (2004).

27 J. Ohe, M. Yamamoto, T. Ohtsuki, and J. Nitta, cond-mat/0409161

28 M. Governale, F. Taddei, and R. Fazio, Phys. Rev. B 68, 155324 (2003).

29 S. A. Tarasenko and N. S. Averkiev, JETP Lett. 75, 552 (2002).

30 X. F. Wang and P. Vasilopoulos, Phys. Rev. B 67, 085313 (2003).

31 G. Usaj and C. A. Balseiro, Phys. Rev. B 70, R041301 (2004).

32 B. Das et al., Phys. Rev. B 39, R1411 (1989).

33 F. E. Meijer et al., cond-mat/0406106

34 A. V. Moroz and C. H. W. Barnes, Phys. Rev. B 60, 14272 (1999).

35 F. Mireles and G. Kirczenow, Phys. Rev. B 64, 024426 (2001).

36 M. Governale and U. Zülicke, Phys. Rev. B 66, 073311 (2002); Solid State Commun. 131, 581 (2004).

37 M. G. Pala, M. Governale, U. Zülicke, and G. Iannaccone, cond-mat/0409580

38 Th. Schäpers, J. Knobbe, and V. A. Guzenko, Phys. Rev. B 69, 235323 (2004).

39 J. Wang, H. B. Sun, and D. Y. Xing, Phys. Rev. B 69, 085304 (2004).

40 M. Cahay and S. Bandyopadhyay, Phys. Rev. B 69, 045303 (2004).

41 Yu. V. Pershin, J. A. Nesteroff, and V. Privman, Phys. Rev. B 69, R121306 (2004).

42 E. T. Jaynes and F. W. Cummings, Proc. IEEE 51, 89 (1963).

43 L. Allen and J. H. Eberly, Optical Resonance and Two-level Atoms, (Wiley, New York, 1975).

${ }^{44}$ M. Pletyukhov and O. Zaitsev, J. Phys. A: Math. Gen. 36, 5181 (2003).

45 S. Debald and C. Emary, cond-mat/0410714

46 R. Arvieu and P. Rozmej, Phys. Rev. A 50, 4376 (1994); ibid. 51, 104 (1995).

47 M. Chaichian and R. Hagedorn, Symmetries in Quantum Mechanics - From Angular Momentum to Supersymmetry (IOP Publishing, Bristol, 1998).

48 L. I. Glazman, G. B. Lesovik, D. E. Khmel'nitskii, and R. I. Shekter, JETP Lett. 48, 239 (1988). 